\title{
Erratum to: Time optimal control of an additional food provided predator-prey system with applications to pest management and biological conservation
}

\author{
P. D. N. Srinivasu • B. S. R. V. Prasad
}

Published online: 22 October 2009

(C) Springer-Verlag 2009

Erratum to: J. Math. Biol. DOI 10.1007/s00285-009-0279-2

The proof of the Theorem 5 in Srinivasu and Prasad (2009) is incomplete as the hypothesis of the theorem does not always imply the inequality (37). It is possible that $1-\xi+\alpha_{\min } \xi \geq 0$. Below we fill this gap by presenting the remaining proof of Theorem 5 when the parameters satisfy

$$
1-\xi+\alpha_{\min } \xi \geq 0
$$

To prove this part we make use of the properties of the zero solution of the linear system (21), (22) which governs the co-state variables along the optimal path. This linear system can be conveniently written in a matrix form as

$$
\left(\begin{array}{l}
\frac{d \lambda}{d t} \\
\frac{d \mu}{d t}
\end{array}\right)=\left(\begin{array}{ll}
-a_{1}(t) & -b_{1}(t) \\
a_{2}(t) & -b_{2}(t)
\end{array}\right)\left(\begin{array}{l}
\lambda(t) \\
\mu(t)
\end{array}\right)
$$

The online version of the original article can be found under doi:10.1007/s00285-009-0279-2.

P. D. N. Srinivasu $(\varangle)$ · B. S. R. V. Prasad

Department of Mathematics, Andhra University,

Visakhapatnam 530 003, India

e-mail:pdns@rediffmail.com

B. S. R. V. Prasad

e-mail: srvprasad_bh@hotmail.com 
where

$$
\begin{aligned}
& a_{1}(t)=1-\frac{2 x(t)}{\gamma}-\frac{(1+\alpha(t) \xi) y(t)}{(1+\alpha(t) \xi+x(t))^{2}} \\
& b_{1}(t)=\frac{\beta(1+\alpha(t) \xi-\xi) y(t)}{(1+\alpha(t) \xi+x(t))^{2}} \\
& a_{2}(t)=\frac{x(t)}{1+\alpha(t) \xi+x(t)} \\
& b_{2}(t)=\frac{\beta(x(t)+\xi)}{1+\alpha(t) \xi+x(t)}-\delta
\end{aligned}
$$

Observe here that $a_{2}(t)>0$. From the assumption (E.1) we have $b_{1}(t) \geq 0$. If $\alpha(t)=\alpha_{\min }$ then from the hypothesis it follows that $b_{2}(t)>0$. Here $a_{1}(t)$ can be either positive or negative depending on the values of the parameters and the state variables.

The characteristic equation associated with the system (E.2) is

$$
m^{2}+\left(a_{1}(t)+b_{2}(t)\right) m+\left(a_{1}(t) b_{2}(t)+a_{2}(t) b_{1}(t)\right)=0
$$

The system (E.2) essentially admits $(0,0)$ as its equilibrium. By studying the qualitative behavior of the system (E.2) based on the properties of the functions $a_{1}(t)+b_{2}(t)$ and $a_{1}(t) b_{2}(t)+a_{2}(t) b_{1}(t)$, we can assess the behavior of the equilibrium solution $(0,0)$. From Theorem 4 in Srinivasu and Prasad (2009), if we assume the value of $\lambda$ at the terminal time $T$ to be positive, from the continuity of $a_{1}(t)+b_{2}(t)$ and $a_{1}(t) b_{2}(t)+a_{2}(t) b_{1}(t)$, it implies that there exists a left neighborhood of $T$, say $[a, T]$, in which we have $\lambda(t)>0$ and $\mu(t)<0$. The proof would be complete if we can show that $a=0$. Below we shall show that it is possible to choose the initial values for the costate variables in such a way that these variables do not change their sign in $[0, T]$, as a consequence the switching function also does not change its sign along the optimal path.

Since $b_{1}(t) \geq 0$, the discriminant of (E.2) can change its sign and consequently, the path of the system (E.2) initiating in the fourth quadrant of $\lambda \mu$-space may leave that quadrant as time progresses. Note that at the terminal time $T$, we have $x(T)=0$ and $y(T)>1+\alpha_{\min } \xi$. Thus the zero solution of the system (21), (22) behaves like a saddle in the vicinity of the terminal time. Therefore, it is always possible to choose the initial value for the costate variable $\mu$ sufficiently far from 0 on the negative $\mu$-axis (with $\lambda(0)>0$ so chosen to make the associated Hamiltonian take the value -1 at $t=0)$ so that by the time the co-state gets closer to positive $\lambda$-axis it is influenced by the the saddle nature of the zero solution. Therefore, the solutions initiating in the fourth quadrant will not leave that quadrant. Therefore we have $\sigma(t)>0$ for all $t \in[0, T]$ and hence $\alpha(t)=\alpha_{\min }$ for all $t \in[0, T]$. 


\section{Reference}

Srinivasu PDN, Prasad BSRV (2009) Time optimal control of an additional food provided predator-prey system with applications to pest management and biological conservation. J Math Biol. doi:10.1007/ s00285-009-0279-2 